\title{
Emergency Biosafety Management Practice in Laboratory of Shelter Hospital $^{*}$
}

\author{
Yu-song LIU1 ${ }^{1}$, Duan-liang PENG ${ }^{1}$, Jia YANG ${ }^{2}$, Dun-yan $\mathrm{CHEN}^{3}$, Hong-bing JIA ${ }^{4}$, Si-yuan YU ${ }^{5}$, Huan-huan CHEN ${ }^{6}$, \\ Kang CHEN ${ }^{2}$, Lyu-rong LIU ${ }^{1 \#}$ \\ ${ }^{1}$ Sichuan National Emergency Medical Rescue Team/Sichuan Academy of Medical Sciences, Sichuan Provincial People's \\ Hospital (East), Chengdu 610100, China \\ ${ }^{2}$ Sichuan National Emergency Medical Rescue Team/Sichuan Academy of Medical Sciences, Sichuan Provincial People's \\ Hospital, Chengdu 610054, China \\ ${ }^{3}$ Fujian Provincial Hospital, Fuzhou 350001, China \\ ${ }^{4}$ China-Japan Friendship Hospital, Beijing 100029, China \\ ${ }^{5}$ Shanghai East Hospital, Shanghai 200120, China \\ ${ }^{6}$ Jiangsu Province Hospital/the First Affiliated Hospital of Nanjing Medical University, Nanjing 210029, China
}

(C) Huazhong University of Science and Technology 2020

\begin{abstract}
Summary: At the end of 2019, the novel coronavirus infection outbroke in Wuhan, Hubei Province. On Feb. 2, 2020, Wuhan, as the worst-hit region, began to build "shelter hospital" rapidly to treat patients with mild illness. The shelter hospital has multiple functions such as emergency treatment, surgical treatment and clinical test, which can adapt to emergency medical rescue tasks. Based on the characteristics that shelter hospital only treats patients with mild illness, tests of shelter laboratory, including coronavirus nucleic acid detection, $\operatorname{IgM} / \operatorname{IgG}$ antibody serology detection, monitoring and auxiliary diagnosis and/or a required blood routine, urine routine, C-reactive protein, calcitonin original, biochemical indicators (liver enzymes, myocardial enzymes, renal function, etc.) and blood coagulation function test etc, were used to provide important basis for the diagnosis and treatment of the disease. In order to ensure laboratory biosafety, it is necessary to first evaluate the harm level of various specimens. In the laboratory biosafety management, the harm level assessment of microorganisms is the core work of biosafety, which is of great significance to guarantee biosafety. As an emergency deployment affected by the environment, shelter laboratory must possess strong mobility. This paper will explore how to combine the biosafety model of traditional laboratory with the particularity of shelter laboratory to carry out effective work in response to the current epidemic.
\end{abstract}

Key words: COVID-19; shelter laboratory; biosafety protection; specimen processing

On 11th February, 2019, the World Health Organization (WHO) officially named various diseases caused by novel coronavirus pneumonia $(\mathrm{NCP})$ as corona virus disease, simplified as COVID-19 ${ }^{[1,2]}$, and this disease quickly spread to the overseas areas ${ }^{[3]}$, which poses a huge threat to the public health security. As the worst-hit region, the number of confirmed cases in Wuhan continued to increase. In order to solve this problem, our country began to construct shelter hospital. In order to strengthen the health emergency rescue and the prevention and control of COVID-19, shelter laboratories are guided to carry out tests related to novel coronavirus to ensure the biosafety of shelter laboratory, according to the Prevention and

Yu-song LIU, E-mail: 18980532562@163.com

\#Corresponding author, Lyu-rong LIU, E-mail: 14650050@, qq.com

*This project was supported by Major Research Projects of Sichuan Health Commission (No. 16ZD026).
Control Plan (third) of NCP Infection from National Health Commission of the People's Republic of China, Technical Guidelines for Laboratory Testing (third) of NCP Infection from National Health Commission of the People's Republic of China, Biosafety Guidelines (second) of Clinical Laboratory for NCP, COVID-19 Protocol (sixth) of NCP Infection from National Health Commission of the People's Republic of China published by National Health Commission of the People's Republic of China, and Guidelines for Laboratory Testing and Biosafety Protection for NCP in 2019 and other files issued by the Chinese Medical Association. Based on the current situation of shelter hospitals, regulations are formulated for biosafety of shelter laboratory.

\section{USAGE AREAS}

These rules are summarized from the construction of shelter hospital in Wuhan, and have great significance 
in performing COVID-19 detection in shelter hospital laboratory.

\section{CHARACTERISTICS OF ETIOLOGY AND EPIDEMIOLOGY}

The novel coronavirus (SARS-cov-2) is the seventh coronavirus isolated from humans, which has the typical characteristics of the coronavirus family and belongs to the $\beta$-coronavirus. The knowledge of the virus mainly comes from the study of SARS-COV and MERS-CoV. The virus is very sensitive to ultraviolet radiation and heat. At a temperature of $56^{\circ} \mathrm{C}$ and a time of $30 \mathrm{~min}$, ethyl ether, $75 \%$ ethanol, chlorinecontaining disinfectant, peracetic acid, chloroform and other lipid solvents can effectively kill the virus. Chlorhexidine cannot effectively inactivate the virus. The incubation period is $1-14$ days, with an average of 5-6 days. Transmission routes include respiratory droplets and close contact in a closed environment and prolonged exposure to high concentrations of aerosols. People generally lack immunity, and almost everyone is susceptible. Some novel coronavirus infections have been reported as asymptomatic or mild ${ }^{[4,5]}$. These patients have strong virus transmission ability ${ }^{[6]}$, and the illness worsens after infection in the elderly and patients with underlying diseases, as well as in children and infants ${ }^{[7]}$. Alveolar lavage fluid is the first choice for nucleic acid detection of patients. However, due to the high risk of operation, throat swabs and nose swabs were recommended to replace the specimens. The blood and other body fluids of patients could not be determined to identify whether they contained virus, so all specimens of patients should be processed according to infectious death processing.

\section{PRINCIPLES OF SAFETY CONSTRUCTION}

\subsection{Basic Requirements for Laboratory Biosafety}

Novel coronavirus infection has been included in the Law of the People's Republic of China on Prevention and Control of Infectious Diseases, as a Class B statutory infectious disease, but doctors prevent and control it on the basis of Class A statutory infectious disease ${ }^{[8]}$. During the clinical observations, the laboratory biosafety level of the shelter hospital should meet biosafety level II standard, and the experimenter must use the personal protection required by biosafety level III laboratory.

\subsection{How to Choose the Place}

Shelter laboratory is an emergency laboratory established according to the scale of the outbreak. In the face of difficulties such as high biosecurity risk, insufficient infrastructure and tight schedule, a temporary molecular biology laboratory is needed to be set up in suitable locations according to local conditions. Temporary molecular biology laboratory can be constructed in the tent, but must meet the standards of biosafety level II laboratory. Partition must be established in the laboratory, biosafety cabinets for sample pre-treatment, incubation, nucleic acid extraction apparatus, such as dry bath pretreatment equipment set in one area and amplification area built in another tent in order to separate as much as possible. A buffer room must be set up in the amplification area to provide the largest biological safety protection for the laboratory area. The laboratory protective clothing can be shared with the medical staff in the cabin but attention must be paid to disinfect themselves when leaving the lab, and then enter the shelter hospital to take off in protective clothing area. The location of the laboratory must take into account the outdoor wind direction and the airflow inside the laboratory, so as to avoid close access to the passage for medical care and patients. In the laboratory, air disinfection instruments are used to purify the air in the laboratory. Meanwhile, all operations must be carried out in the biosafety cabinet to avoid biological exposure caused by laboratory airflow. Involving other related tests, including complete blood count, liver and kidney function and biochemical examination, etc., can be conducted in other separate temporary tents, the buffers, or the corresponding equipment, and the laboratory must be equipped with biosafety cabinet and UV lamp, etc. The pretreatment of biosafety equipment specimens should be guaranteed to take place in biosafety cabinets to prevent biological exposure.

\section{BIOSAFETY PROTECTION}

\subsection{Protective Equipment Management}

Shelter hospital faces patients who are already diagnosed with coronavirus infection, medical care personnel can choose protective equipment like medical surgical masks (YY0469-2011), medical respirator (GB19083-2010), the N95 (American NIOSH42CF R84-1995, the Chinese KN95 [GB26262019]), comprehensive types of respiratory protection device, protective clothing, clothing, overalls, latex gloves, medical helmets, goggles, facing screen and shoes. Wearing and taking off protective equipment must be carried out in the designated place of shelter hospital. Every step of wearing and taking off must be supervised and inspected by a special person.

\subsection{Level of Biosafety Protection}

Shelter hospital including lab personnel must receive Level III protection (Biosafety Level 3 protection: medical respirator, double latex gloves, face screen/goggles, protective clothing over work clothes, medical helmet, boot cover, and hand hygiene). All tests involved during work must be performed in the 
biosafety cabinet.

\subsection{Ultraviolet Rays}

Laboratories must be equipped with ultraviolet disinfection lamps, and regularly test the performance of ultraviolet lamp, complete record. The accumulative disinfection time of ultraviolet lamp does not exceed the service life of ultraviolet lamp. Within the prescribed time limit, each disinfection time is not less than $30 \mathrm{~min}$.

\subsection{Disinfectant}

Before and after work, $500 \mathrm{mg} / \mathrm{L}$ effective chlorine disinfectant (100 times diluted 84 disinfectant) or $75 \%$ ethanol IS should be used for table and floor disinfection.

\subsection{Hand Hygiene}

Shelter hospital is rebuilt for emergency use, so routine hands hygiene can not be achieved. Shelter hospital includes laboratories that all use non-detergent for hand hygiene, but the procedures are the same as the method of hand washing with running water (sixstep washing technique). Be careful not to touch the skin of the face, touch the eyes and pick the nose etc. It is recommended not to wear long hair or accessories.

\subsection{Specimen Collection}

The subjects are confirmed COVID-19 cases, and the test items include blood routine, whole blood CRP, liver and kidney function, lung function test, novel coronavirus serology and nucleic acid test. The collectors shall be protected by Biosafety Level III. The process of collecting specimens shall be safe and targeted, minimizing the contact, avoiding aerosols and droplets and reducing bedtime if possible. When the patient independently completes the collection, such as the sputum and feces specimens, the doctor or nurse will avoid the collection after giving instructions to the doctor.

\subsection{Transportation and Receipt of Specimen}

In the actual work, the specimen collector is required to have Level III protection. After the collection is completed, the specimen collector should be sealed with a sealed bag immediately, and placed into a doublelayer transfer box, which is then put into the designated handover place. Transporters need Biosafety Level II protection (medical respirator, latex gloves, isolation clothing over overalls, medical protective cap). When specimens are received, disinfectant should be on the outside of the transfer box for transportation. During the transfer process, the transfer box should not be opened and be guaranteed that it does not vibrate violently. After the specimens arrive at the safety laboratory, the laboratory staff shall conduct Biosafety Level III protection, open the transfer box in the Biosafety cabinet, take out the specimens from the sealed bag, and spray $75 \%$ ethanol on the surface of the container to sterilize. The signature of each sample is completed through the registration of a hand-held system exclusive to the square cabin. If the sample is signed on paper, the recorded paper cannot be taken out of the laboratory and should be treated as a contaminant. When it is received, the specimen identification information should be confirmed. If there is no identification, the specimen will be rejected, and the unqualified specimen will be treated as discarded specimen. After completion, the transfer box is returned to the designated location for external disinfection. All pretreatment must be conducted in a biosafety cabinet, and the detection personnel should carry out Level III protection. In case of emergency such as specimen leakage during transportation, we must cooperate with the staff of shelter hospital to deal with it and keep a record.

\subsection{Specimen Preparation}

Biosafety Level III protection is required for the laboratory staff before they do the experiments. The specimens of closed tube operation items such as blood routine and coagulation, do not need to be processed and can be directly tested on the machine. Blood samples that need to be centrifuged for other biochemical, immunological and other projects must be put in and taken out of the centrifuge rotor in the biosafety cabinet. During centrifugation, the operator shall not leave the centrifuge. The centrifuge can not be removed until $15 \mathrm{~min}$ after the centrifuge stops centrifuging. The operator must disinfect and open the specimen in the biosafety cabinet. If there is any abnormal sound during centrifugation, centrifugation should be stopped for above $30 \mathrm{~min}$ and then cover is opened and disinfected. The respiratory tract specimens detected by novel coronavirus nucleic acid are treated with dry bath inactivation at $56^{\circ} \mathrm{C}$ for $30 \mathrm{~min}$. Only after the sample tube is cooled to room temperature, could the cover in the biosafety cabinet be opened.

\subsection{Basic Principles of Working}

In shelter laboratory, all tests related to novel coronavirus must be carried out in the spaceindependent Biosafety Level II laboratory, and the operator shall be protected by the Biosafety Level III laboratory. During the test operation, if possible, the opening of the tube cover should be avoided. The duration of opening should be shortened, and the generation of aerosol should be avoided. If the lid must be opened, the operation may generate aerosols, or the specimen itself may be exposed, it must be carried out in the biosafety cabinet and the lid must be discarded for disinfection. The examiners should hold clinical test qualification certificates for blood routine, CRP, biochemical and immunological test operations and results. In addition, personnel conducting nucleic acid testing for novel coronavirus must also participate in clinical gene amplification training and obtain employment certificates.

\subsection{How to Dispose Specimen and Related Waste after Inspection}

If specimens such as blood need to be reviewed, 
new specimens should be created in the biosafety cabinet and then stored in accordance with regulations. Otherwise, they should be packed into red garbage bag and sealed with other solid wastes, and then transferred to shelter hospital for unified treatment. Relevant waste liquid shall be chemically or physically disinfected, and the disinfection effect shall be verified to ensure complete inactivation. After the treatment, they must be transferred to shelter hospital for unified follow-up treatment.

\subsection{Biosafety Accident Handling}

Novel coronavirus specimen contamination of the biosafety cabinet caused limited pollution: the effective chlorine content of the disinfectant is $3000 \mathrm{mg} / \mathrm{L}$. The disinfectant should be prepared and used within $24 \mathrm{~h}$. Laboratory pollution: keep the laboratory space closed, avoid the entrance and exit of irrelevant personnel, and avoid the spread of pollutants. The contaminated area should be covered with towel of $3000 \mathrm{mg} / \mathrm{L}$ effective chlorine disinfectant. If necessary (in case of large overflow), peracetic acid can be used to heat the fumigation laboratory with $2 \mathrm{~g} / \mathrm{m}^{3}$ dose overnight; Or $20 \mathrm{~g} / \mathrm{L}$ peracetic acid disinfectant with aerosol should be sprayed with dosage of $8 \mathrm{~mL} / \mathrm{m}^{3}$ for about $1-2 \mathrm{~h}$. The indoor humidity during fumigation is $60 \%-80 \%$. The pollutants should be cleaned up strictly to prevent secondary hazards according to the requirements of biosafety operation, laboratory ventilation, etc.

\section{DISCUSSION}

Shelter hospital is a major accomplishment of our country in this period, as well as a major breakthrough of our country in public health prevention and control and medical treatment ${ }^{[9]}$. The construction of shelter hospital encounters many difficulties and problems, and its biosafety hazard level is high. In the construction work of shelter hospital in Wuhan, how to do a good job in the biosafety protection of the laboratory is not only responsible for the patients, but also for our staff, as well as for the current epidemic. The essence of scientific research is to benefit mankind, but science is also a double-edged sword, if attention is not paid to basic safety norms, it may bring disaster to mankind. According to the latest statistics released by Johns Hopkins University, nearly 650000 COVID-19 cases and 30249 deaths have been confirmed worldwide as of 15:30 (03:30 GMT) on 28th in the east of America. Furthermore, most cases occurred in the United States, with more than 115000 cases; while Italy has the largest number of deaths, with 1023 people $^{[10]}$. At present, all countries in the world are fighting the epidemic together, so we need to make great efforts to ensure the biosafety protection of every treatment link involved in order to avoid the negligence of biosafety protection that leads to the infection of medical staff.
The inspection group of Sichuan emergency medical rescue team has experience in shelter laboratory construction. Combining previous earthquake rescue experience with the actual situation in Wuhan, this team explores the suitable shelter hospital laboratory biosafety management experience and hopes to share the experience with other teams. Due to the short time, there must be many problems and shortcomings, but our goal is clear, that is, to make the emergency shelter hospital laboratory the most perfect, to avoid our staff infection, and to provide more efficient diagnosis and treatment data supports for patients confirmed with COVID-19.

\section{Conflict of Interest Statement}

The authors declare that there is no conflict of interest in this study.

\section{REFERENCES}

1 National Health Commission. National Health Commission on the new coronavirus pneumonia tentative name Notice of the matter (national health service circular no. Available at: http://www.chinacdc.cn/jkzt/crb/zl/ szkb_11803/jszl_11815/202002/t20200209_212395. Accessed: 7 Feb 2020.

2 WHO. WHO Director-General's remarks at the media briefing on 2019-nCoV on 11 February 2020. Available at: https://www.who.int/dg/speeches/detail/who-directorgeneral-s-remarks-at-the-media-briefing-on-2019-ncovon-11-february-2020. Accessed:11 Feb 2020.

3 WHO. Novel coronavirus (2019-nCoV): situation report-10, Geneva: World Health Organization, 2020. Available at: https:// www.who.int/docs/default-source/ coronaviruse/situation-reports/20200130-sitrep-10ncov.pdf. Accessed:30 Jan 2020.

4 Qiu J. Covert coronavirus infections could be seeding new outbreaks. Nature, 2020. doi: 10.1038/d41586-02000822-x

5 Nishiura H, Kobayashi T, Suzuki A, et al. Estimation of the asymptomatic ratio of novel coronavirus infections (COVID-19). Int J Infect Dis, 2020,94:154-155

6 Zou L, Ruan F, Huang M, et al. SARS-CoV-2 Viral Load in Upper Respiratory Specimens of Infected Patients. N Engl J Med, 2020,382(12):1177-1179

7 Chinese Society of Laboratory Medicine. Chin J Lab Med, 2020,43(00): E001-E001. Available at: http:// rs.yiigle.com/m/yufabiao/1180119.htm?from=groupme ssage\&isappinstalled=0. Accessed:5 Feb 2020.

8 National Health Commission. Prevention and treatment of infectious diseases in the People's Republic of China. Available at: http://www.nhc.gov.cn/fzs/s3576 /201808/6d00c158844f42c5bcf94993bffa665a.shtml. Accessed:29 Jun 2013.

9 Zhang X, Zhou W, Liao J, et al. Xinhua News Agency. Available at: http://www.81.cn/gnxw/2020-02/20/content 9746703.htm. Accessed:20 Feb 2020.

10 Xiao X. Xinhua News Agency. Available at: http:// www.xinhuanet.com/world/2020-03/29/c_1125782643. htm. Accessed: 29 March 2020.

(Received Apr. 30. 2020; accepted Aug. 3, 2020) 